Article

\title{
Anthropogenic and environmental strain on beach environments retrieved and monitored by spaceborne synthetic aperture radar
}

\author{
Valeria Di Biase $^{1 *(D)}$ and Ramon F. Hanssen ${ }^{2}$ (D) \\ 1 Delft University of Technology, 2628 CN, Delft, The Netherlands; v.dibiase@tudelft.nl \\ 2 Delft University of Technology, 2628 CN, Delft, The Netherlands; r.f.hanssen@tudelft.nl \\ * Correspondence: v.dibiase@tudelft.nl
}

Citation: Di Biase, V.; Hanssen, R.F. Preprints 2021, 1, 0. https://doi.org/

Received:

Accepted:

Published:

Publisher's Note: MDPI stays neutral with regard to jurisdictional claims in published maps and institutional affiliations.

\begin{abstract}
Environmental effects and climate change are lately representing an increasing strain of the coastal areas which topography strongly depends on these conditions. However, the processes by which weather and environmental phenomena influence the highly variable beach morphology are still unknown. A continuous monitoring of the beach environment is necessary to implement protection strategies. This paper presents the results of an innovative study performed on a coastal area using satellite remote sensing data with the aim of understanding how environmental phenomena affect beaches. Two-years of synthetic aperture radar (SAR) Sentinel-1 images are used over a test area in Noordwijk, the Netherlands. At the same time as the SAR acquisitions, information on tidal and weather conditions are collected and integrated from nearby meteorological stations. Dedicated codes are implemented in order to understand the relationship between the SAR amplitude and the considered phenomena: wind, precipitation, tidal conditions. Surface roughness is taken into account. The results indicate a strong correlation between the amplitude and the wind. No particular correlation or trend could be noticed in the relation with the precipitation. The analysis of the amplitude also shows a decreasing trend moving from the dry area of the beach towards the sea and the correlation coefficient between the amplitude and the tide level gets negative with the increase of the water content.
\end{abstract}

Keywords: SAR, Sentinel-1; Amplitude; Beach environment; Weather conditions

\section{Introduction}

In the last decades, anthropogenic activities, environmental effects, and climate change are representing a increasing strain on the coastal environment, resulting in ecological, economical, societal, and safety concerns. Commerce, transportation, tourism and fishing are among the economical services affected by these factors [burkett2012coastal]. With its highly dynamic morphology, the beach environment strongly depends on atmospheric conditions both in short and long-term [wright2015ocean], as well as human activities. However, the processes by which storm, wind, precipitation, tides, currents, and human activities influence coastal morphology, in particular erosion, have not been fully investigated.

Continuous monitoring of the coastal area, including its topographic and morphological state, is of great importance for a better understanding and to implement protection strategies able to adapt to climate change [klemas2009role, bochev2011decadal, keijsers2015adaptation, benveniste2019requirements]. Ground-based surveying is currently able to provide accurate measurements with a resolution of a few centimeters both for coastal and nearshore areas [mason2000beach, mielck2014high, porskamp2018multiscale], but it is not efficient for the evaluation of big areas due to logistical difficulties and high costs [choi2018optimum, salameh2019monitoring]. Spaceborne remote sensing techniques provide a viable alternative tool for topography and bathymetry mapping of coastal areas [salameh2019monitoring]. For a better understanding of topography variation, particular consideration shall be given to soil moisture. Surface moisture, governed by complex tidal and weather phenomena, groundwater and capillary flow [atherton2001inter, 
namikas2010temporal] is in fact one of the drivers of coastal dune development affected by aeolian transport [davidson2005effect, bauer2009aeolian, ellis2012temporal, de2014aeolian]: it increases the threshold wind speed at which sediment begins to move, by increasing the resistance of the upper sand layers against wind erosion [mckenna1989theoretical, cornelis2003effect]. It is affected by many factors such as tides, precipitation, temperature, and soil characteristics, and it can be considered as an indicator of environmental changes (such as long-term shoreline changes, dust storms, or erosions) [ahmed2011review].

Traditional soil moisture observations on coastal areas include direct in situ measurements [wiggs2004dynamic, davidson2005effect, yang2005rapid, nield2014detecting]. Unfortunately, in situ measurements are time-consuming and expensive, very localized in scale, and impractical for a wide coverage due to the high spatial variability of the target. Remote sensing, with its ability to detect soil moisture, provides a unique ability to monitor and map coastal areas with complete, repeated, and frequent coverage of the Earth's surface. Optical remote sensing techniques are based on the principle that wet sand is less reflective than dry sand, due to pore water surrounding the sand grains [nolet2014measuring]. Their potential for aeolian research was demonstrated by [mckenna2006measurement, darke2008field, delgado2009application] and while widely used, these techniques are susceptible to cloud, rain, and light conditions. This is not convenient in areas with high average annual cloud cover. Active microwave remote sensing systems, despite their lower resolution, can penetrate through clouds and provide continuous and all-weather monitoring. This allows for more reliable and consistent sand monitoring. Synthetic Aperture Radar (SAR) is the most common active remote sensing system for Earth observation [csekertekin2018soil]. In recent years, many studies demonstrated the advantage of using SAR for the estimation of soil surface characteristics, such as surface roughness and soil moisture [baghdadi2002retrieving, srivastava2009large, zribi2014new, gorrab2015potential]. Different sensor configurations, in terms of wavelength, polarization, and incidence angle, allow for the discrimination of various soil parameters, such as surface roughness, soil dielectric constant, and vegetation cover [fung2002improved, attarzadeh2018synergetic]. The exploitation of X-, L-, or C-band for soil moisture retrieval has been presented in many studies: from X-band [aubert2011analysis, baghdadi2011estimating, zribi2011soil], to C-band [zribi2003new, balenzano2010dense, lievens2012spatial, jacome2013monitoring] and L-band [paloscia2012com kim2013models, wigneron2017modelling].

SAR backscatter is directly related to the dielectric constant of the target [elachi87], which describes the electrical properties of a medium relative to the dielectric constant of free space: for water, the (dimensionless) dielectric constant is $\sim 80 \%$, and for dry soil it is $\sim 2 \%$ [moran2000soil]. This strong contrast between the dielectric properties of water and dry soil forms the base of microwave soil moisture estimation. However, the backscatter is also a function of target-specific factors such as the roughness of the ground surface. Several semi-empirical models for estimating and disentangling soil moisture and surface roughness from radar backscattering coefficients have been reported in the literature [dubois1995measuring, smith2004rough], but none of these models has been tested on sandy areas. One of the most common characterizations of surface roughness is its root-mean-squared height (RMSH). For this purpose, Terrestrial Laser Scanning (TLS) has been investigated for its huge potential for coastal processes [di2021sensitivity, french2009coastal], as it allows fast and repeatable measurements of surface topography [nield2014detecting] and surface moisture. Several studies demostrated how soil roughness can be evaluated using TLS [perez2007modeling, haubrock2009spatiotemporal]

A further advantage of the use of SAR systems for understanding of the beach environment and aeolian transport is the possibility to provide knowledge of the characteristics of wind fields over both the ocean and the littoral zones with high spatial resolution [huang2017technical]. Satellite active microwave sensors appear to be the best instruments for information about wind over large offshore areas [zecchetto2010ocean, guo2018assessing]. Many approaches for wind retrievals have been investigated, e.g. 
[wackerman2002two, hersbach2003cmod5, yang2011comparison, liu2013systematic] for C-band co-polarized SAR images. The backscatter coefficient $\sigma_{0}$ for cross-pol (cross polarization) linearly increases with wind speed without saturating [hwang2010comparison, zhang2015synergistic, zhang2017hurricane], gradually transiting to a cubic curve for high wind condition $(>10 \mathrm{~m} / \mathrm{s})$, whilst for co-pol $\sigma_{0}$ increases and saturates for wind speed higher than $16 \mathrm{~m} / \mathrm{s}$ [hwang2010comparison]. Sentinel-1 VV and HH SAR images for wind field retrievals have been investigated by [zecchetto2016wind] and [monaldo2016preliminary].

Here we present the results of a study performed on a beach at Noordwijk, the Netherlands, located central on the Dutch coastline. The dataset includes a two-years stack of Single Look Complex (SLC) Sentinel-1 images, VH and VV polarization, with a six-day temporal resolution, all taken from the same descending orbit parallel to the coastline. We study the potential of SAR for the retrieval and monitoring of soil properties over a sandy area, to search forpotential empirical relationship between the SAR amplitude and wind, precipitation and tidal condition (affecting the soil moisture). Human activities have not been considered in the present work. Soil roughness, in terms of RMSH, has been retrieved using a dedicated TLS system. In Section 2 the study area, the satellite imagery, and the ancillary data are presented, while Section 3.2 evaluates the amplitude analysis in terms of mean and range. Section 3.3 shows the analysis of the wind conditions, Section 3.4 presents the results of the rain conditions, while Section 3.5 reports the investigation of the tidal condition. In Section 3.6 the roughness of the study area and its correlation with the SAR signal have been evaluated.

\section{Data collection}

\subsection{Study area}

The study site is a beach section at Noordwijk, located at $52.24 \mathrm{~N}, 4.42 \mathrm{E}$. The area investigated covers a surface of about $100 \mathrm{~km}^{2}$. A large part of the beach $(\sim 130 \mathrm{~m}$ wide) consists of a dune area covered by vegetation. The dune area has not been taken into account for the analysis considering the presence of vegetation and the highly variable slopes which require a different approach for the investigation of the radar signal. The considered area is relatively narrow ( $\sim 140 \mathrm{~m}$ wide in case of low tide) and heterogeneous, consisting predominantly of fine quartz sand (>90\%) [eisma1968composition]. We estimated the mean grain size to be $\sim 260 \mu \mathrm{m}$ using sieving methods from sediment collected within the top $2 \mathrm{~mm}$. Fieldwork measurements were taken in order to estimate the shoreline position in case of high tide and low tide. These measurements have been used for the mask evaluations in Section 3.1.

\subsection{Satellite imagery and pre-processing}

The considered Sentinel-1 dataset collected over the Dutch coast ranges from January 2018 until December 2019. The SAR data are available both in VV and VH polarization. A total of 106 and 108 images from a descending orbit (DSC110) has been used for VH and VV, respectively. The dataset, provided in Interferometric Wide swath mode (IW), consists of Level-1 Single-Look Complex (SLC) products, where each image pixel is represented by a complex value and contains both amplitude and phase information. The imagery is geo-referenced using orbit and attitude data from the satellite. Each IW SLC image consists of a series of bursts. The acquisition orbit selected was descending, parallel to the coastline, see Figure 1, to ensure optimal coverage of the area of interest.

IW SLC products are re-sampled to a common pixel spacing grid in range and azimuth. The pre-processing of the satellite imagery has been done by using the SNAP tool ${ }^{1}$. The data were split by selecting the subswath, the burst and the polarization, and then coregistered. After co-registration, a subset containing the area of interest was cropped. For each pixel, the amplitude has been automatically evaluated as a function of the given complex number. We decided not to apply any sort of noise correction or mitigation on

\footnotetext{
https://step.esa.int/main/toolboxes/snap/
} 


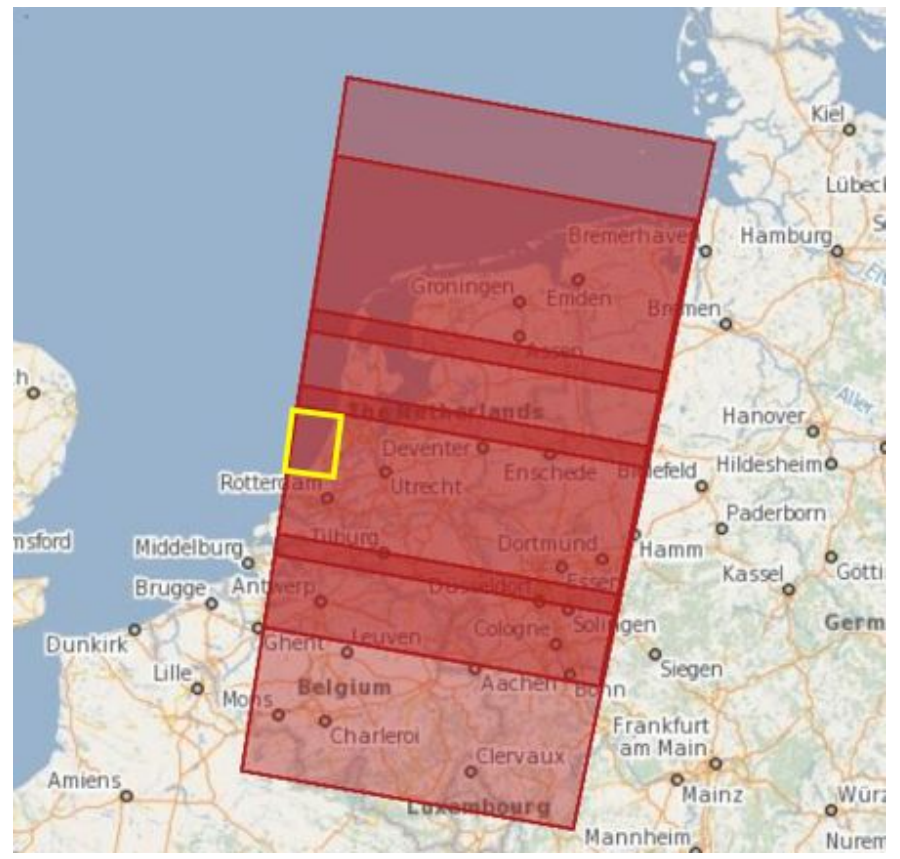

Figure 1. Footprint of the selected descending Sentinel-1 (DSC37) over the Netherlands, with the study area highlighted.

the amplitude value in order to keep the original information. In order to have amplitude values easily comparable in the figures, given the amplitude $A=\sqrt{i^{2}+q^{2}}$, the amplitude values used in this paper are obtained as $\log _{10}(A)$

\subsection{Ancillary Data}

Numerous meteorological stations continuously monitor the area of interest providing detailed variables. For the present study, information regarding wind (speed and direction) and precipitation accumulation at the time of the satellite pass were collected. Four weather stations were selected since they provide the type of data and the temporal sampling required: two amateur and two professional meteorological stations. The amateur meteorological stations are located in Noordwijk (52.25N, 4.43E) and Katwijk (52.19N, $4.41 \mathrm{E}$ ), both on the beach and close to the area of interest (respectively $1 \mathrm{~km}$ and $7 \mathrm{~km}$ from the area of interest); the professional weather stations ${ }^{2}$ are located at IJmuiden $(52.463 \mathrm{~N}, 4.555 \mathrm{E})$ and Schiphol $(52.318 \mathrm{~N}, 4.790 \mathrm{E})$, i.e., $31 \mathrm{~km}$ and $34 \mathrm{~km}$ from the area of interest, respectively. The datasets of the two KNMI weather stations have high correlation coefficients with each other concerning the wind and rain variables. The wind data set of station Noordwijk during the two years is not as complete as station Katwijk, while the opposite goes for the rain dataset, where the station in Katwijk does not contain a fully complete dataset. The two amateur weather stations at Katwijk and Noordwijk are closely correlated with the KMNI stations and therefore they have been used as a reliable source for wind (speed and direction) and precipitation accumulation. Tidal conditions information are provided by the Dutch authorities (Rijkswaterstaat) and freely accessible online $^{3}$. As for the weather conditions, tidal conditions at the time of the satellite pass have been considered.

2 KNMI: Koninklijk Nederlands Meteorologisch Instituut, https://www.knmi.nl/nederland-nu/klimatologie/daggegevens

3 https://www.worldtides.info/copyright 


\section{Data Analysis}

\subsection{Masks selection}

In the subset cropped at the end of the SAR pre-processing, six masks have been classified depending on the coverage as shown in Figure 2. The analysis of the amplitude has been performed per mask. The six masks include: (i) a city area, which has been mostly

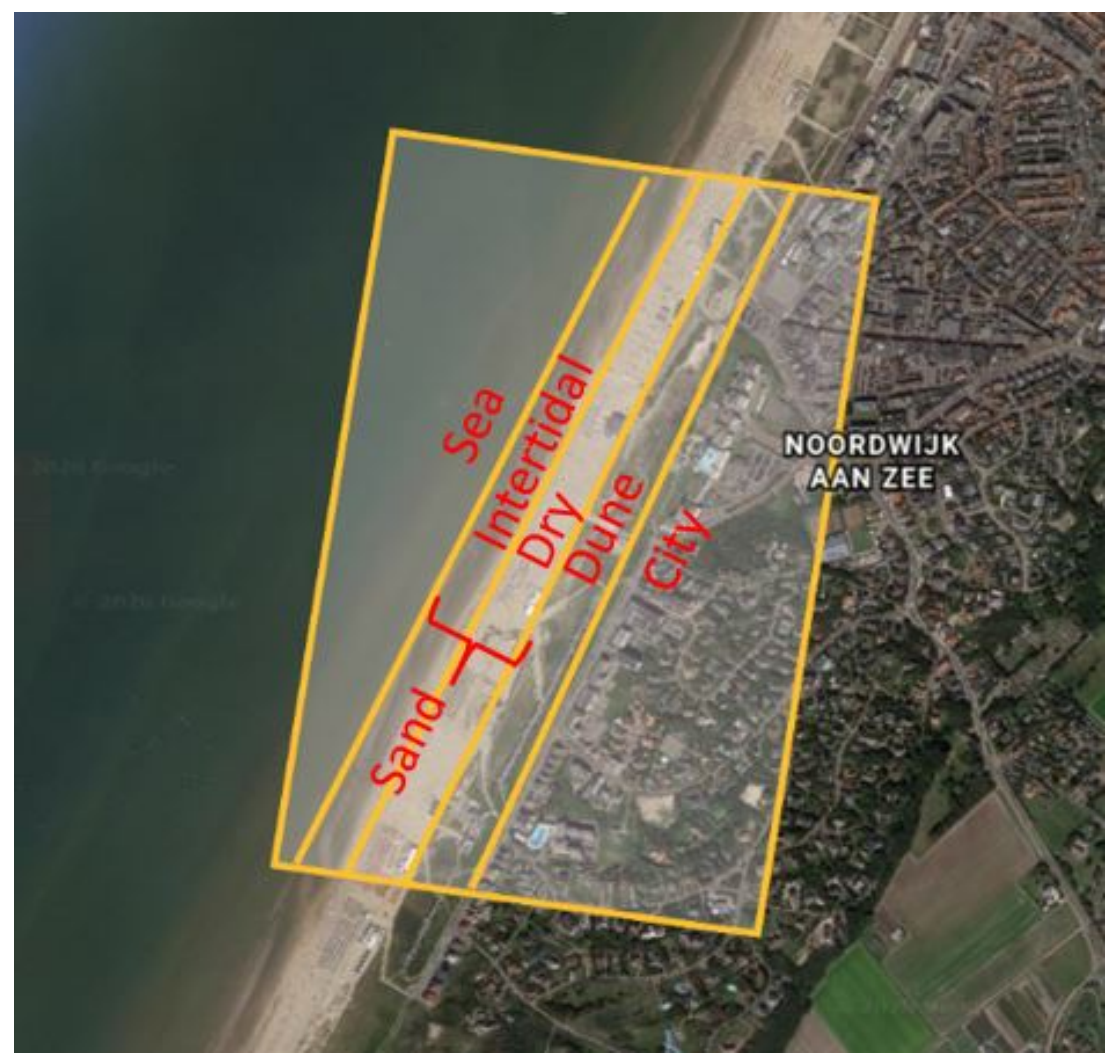

Figure 2. Masks selected on the study area.

used for evaluating the low-variability pixels and for data calibration; (ii) a dune area, including the dunes and vegetation zone located between the city and the beach, which has not been evaluated due to the complexity of the area (including slope and vegetation) and to its limited relevance for the present study; (iii) the dry part of the beach, i.e. the area not affected by tidal cycles but only by weather condition and human activities. This area also includes small constructions such as bars, restaurants, temporary stands, or boats permanently or temporary located on the beach; (iv) the intertidal area, which is the area affected by the tides and which can be either partially dry or covered by sea depending on the tidal condition; (v) the shallow sea area, just behind the main surf where waves are breaking, particularly relevant for the analysis of the wind conditions. Finally, for some of the analyses the union of the dry and the intertidal area masks is considered as an additional mask. This (vi) mask includes therefore the entire sandy area.

\subsection{Amplitude Calibration}

The SAR signal is typically affected by soil properties with the same order of magnitude as the signal wavelength [ulaby1986microwave], i.e. the surface roughness, and the dielectric properties and water content. We performed a sensitivity analysis to understand how the amplitude of Sentinel-1 from a coastal area is affected by the above mentioned parameters. As a first step, an empirical local calibration of the images has been done. The city mask showed the least amplitude variability over time. Within this mask, the $25 \%$ of the pixels with the least amplitude variability (smallest standard deviation) over time have been selected and used for calibrating the images. This calibration has been done separately for the VV and the VH polarization. For the VH polarization, the mean ampli- 
tude over time for the selected pixels ranges between $0.92-1.13$, whilst for VV polarization it ranges between 0.75-1.11. Figure 3 (left), for VV polarization, shows that there is no significant correlation $(r=0.25)$ between the amplitude values of the city mask and the ones of the sand mask (e.g. caused by noise in the image). Figure 3 (right) shows instead a high correlation $(r=0.84)$ between the sand and sea masks. The colorbar expresses the wind intensity. For VV polarization, the correlation factor between city and sand mask is $r=0.05$ and between the sea and sand it is $r=0.68$. Figure 3 also shows that there is no clear correlation between the wind speed and the city mask, whilst the wind speed shows a more explicit influence on the sand and sea areas.
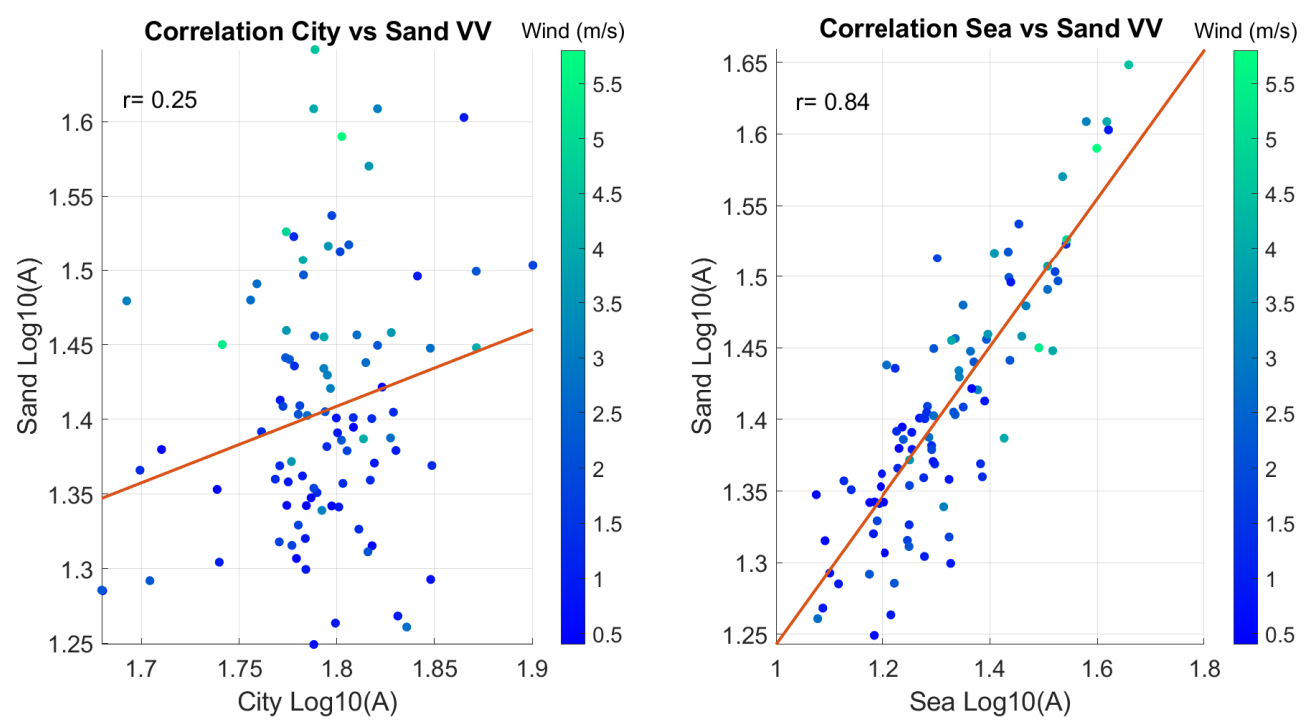

Figure 3. Correlation factor between pairs of masks. The colorbar represents the wind intensity. Left: correlation factor between city and sand masks (VV polarization). Amplitude values of the city area and of the sand area are poorly correlated. Right: a high correlation occurs between the sand and sea masks. The hypothesis is that they are influenced by phenomenon, i.e., wind. As the colorbar shows, there is not clear correlation between environmental factors (such as wind) and the city mask, whilst the wind intensity has a more relevant influence on the sand and sea masks.

After calibrating all the images relatively to the low variability area, we analyze the mean amplitude value of the considered masks as a function of time for both the polarizations VV and VH, see Figure 4. The highest values occur for the city mask, which is also shows the least variability (13\% over time) and the least influenced by the weather conditions.

The maximum variability (31\%) and the lowest mean values occur in the shallow sea area. We hypothesize that is that this is mainly due to the wind, which affects the surface roughness. A high variability (20\%) also occurs in the sandy area. This can be due to anthropogenic factors (e.g. the presence of boats or temporary stands during summer, people, tractors, sand deposits built during winter around the buildings, the effect on the moisture and on the roughness of the tide), weather condition (the wind reshaping the sand), and tidal conditions. For VH polarization, similar conditions occur but the variability of the three masks is lower: City, 6\%; Sand, 15\%; and Sea, 11\%. Thus, it appears that VV polarization is more sensitive to phenomena affecting both the sand and the sea mask. Figure 4 also represents VV and VH ranges in the city, the sandy and the shallow sea mask. It shows how this maximum value decreases going from the city towards the sea. The same situation occurs for $\mathrm{VH}$, but its values always range lower than VV.

This analysis also showed that it is not possible to identify a temporal or a seasonal trend in the amplitude values. 

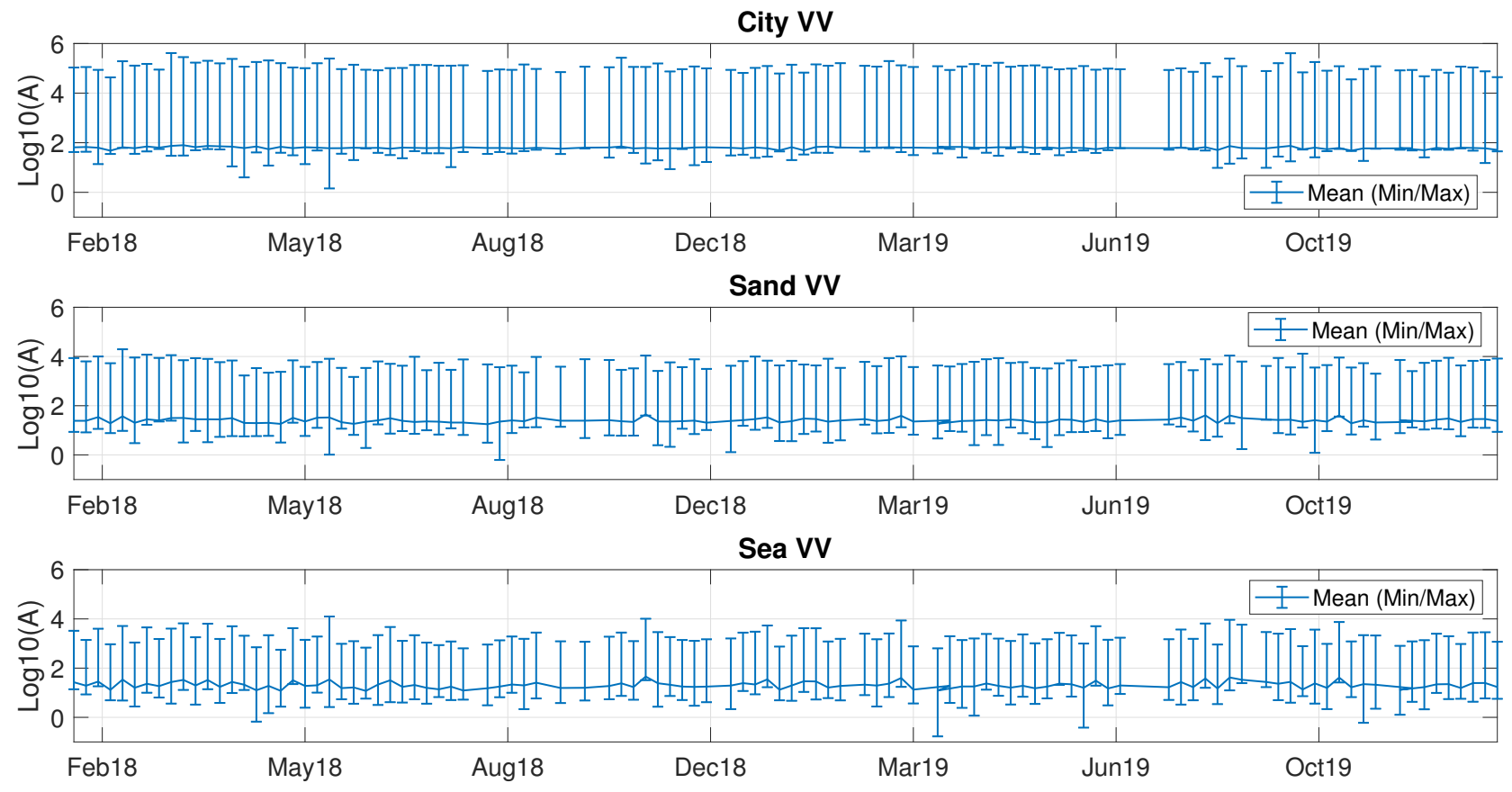

Figure 4. Comparison between ranges and mean amplitude values over 2-years for the city (first raw), sand (second raw), and sea (third raw) masks (VV polarization). The highest values occur for the city, which is also the area with the smallest mean amplitude variability over time. The maximum variability and the least mean values occurs for the sea area, showing a decreasing trend of the amplitude moving from the city towards the sea. High variability both in the range and in the mean values also occurs in the sandy area. This can be due to anthropogenic activity, weather conditions, and tidal conditions. 


\subsection{Wind analysis}

To investigate the sensitivity of the amplitude signal to the wind, scatter plots of the amplitude at $\mathrm{VV}$ and $\mathrm{VH}$ polarization versus wind intensity and speed were generated. Figure 5 shows respectively the plots of the wind speed and direction as a function of the amplitude mean value for the sand and sea masks in both VV and $\mathrm{VH}$ polarizations. The amplitude mean value is shown in the colorbar, in a logarithmic scale. A dependence of amplitude on the wind direction can be noticed both in the two masks and in the two polarization.

Considering the sand mask, the distribution of wind intensity is associated to low or high amplitude depending on the wind direction. For some wind angle (i.e., $210^{\circ}-240^{\circ}$ ), the dependence of the amplitude on the wind is more considerable. The same considerations can be done for the sea mask, where it can be noticed how low amplitude values are associated with low wind intensity, which increases going towards higher amplitude values. A dependence from the wind direction can be also noticed, e.g. in the range $270^{\circ}$ $360^{\circ}$. In general, for both polarizations and both masks, a high wind intensity is associated with high amplitude values.

The correlation factor between the amplitude and the wind intensity has been evaluated for both polarizations and for all the masks. The highest correlation occurs obviously in the sea mask for both polarizations, with a correlation factor $r=0.56$ for $\mathrm{VH}$ and $r=0.62$ for VV. Polarizations VV and VH also show a significant correlation on the sand area, where the correlation factors are $r=0.57$ and $r=0.51$ respectively, see Figs. $6 \mathrm{a}$ and $\mathrm{b}$.

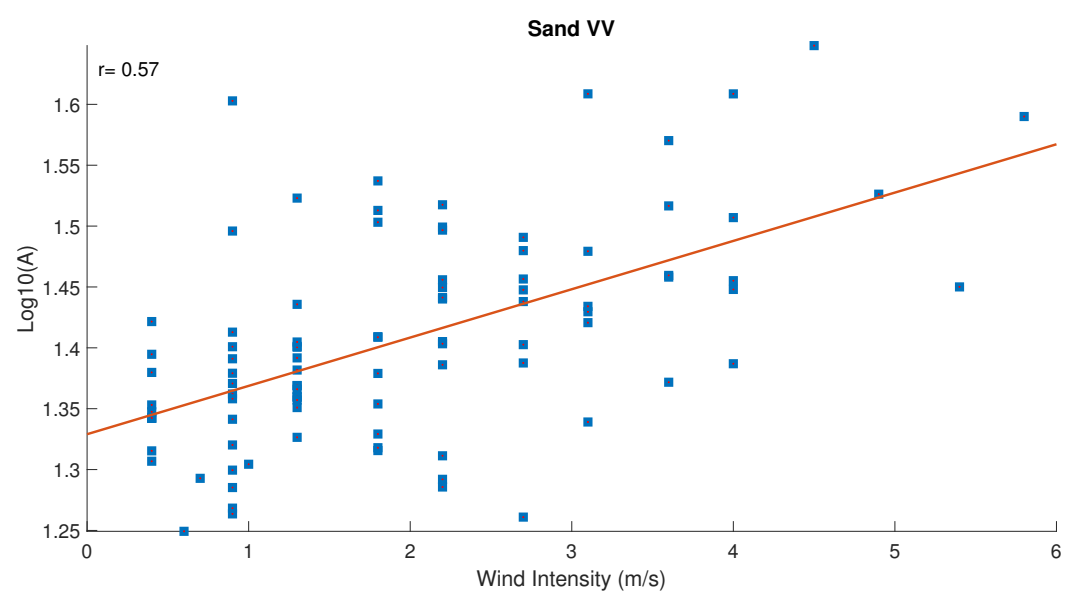

(a)

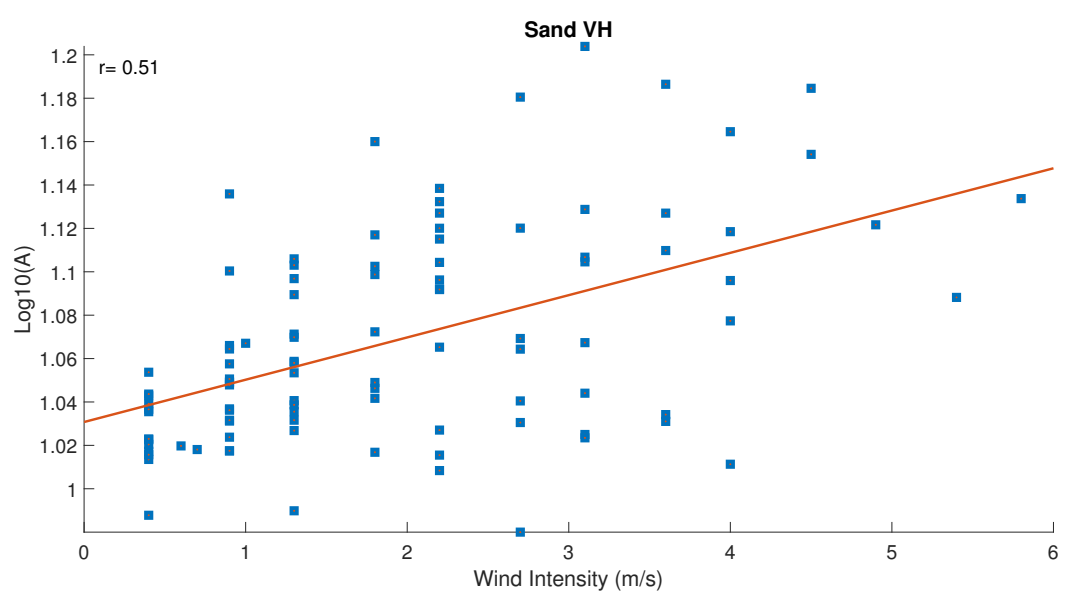

(b)

Figure 6. Correlation factor between wind intensity and amplitude on the sand mask for VV (a) and $\mathrm{VH}$ (b) polarization. 


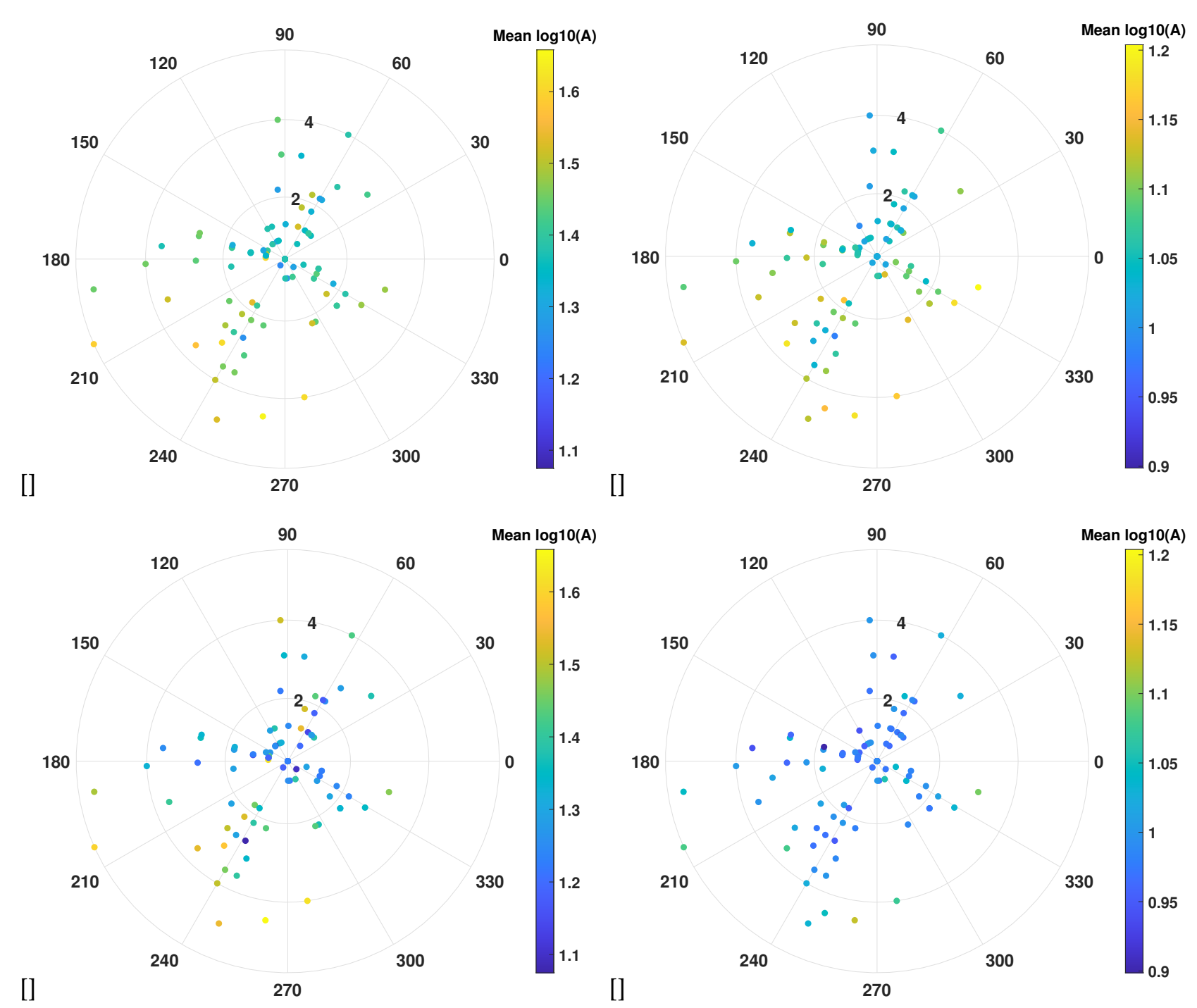

Figure 5. Amplitude in logarithmic scale (color bar) as a function of the wind direction $\left(0^{\circ}=\right.$ North; $90^{\circ}=$ East $)$ and wind intensity ( $\mathrm{m} / \mathrm{s}$; represented on the radius). Amplitude in VV and VH polarizations is represented, respectively, on the sand mask in the panels (a) and (b), and on the sea mask in the panels (c) and (d). A dependence of amplitude on the wind direction can be noticed both in the two masks and in the two polarization. In general, for both polarizations and both masks, high wind intensity is associated with high amplitude values. For some wind angle (i.e. $210^{\circ}-240^{\circ}$ ), the dependence of the amplitude on the wind is more considerable. 
It is interesting to note that the correlation increases or decreases depending on the wind direction. Figure 7 shows how the correlation factor has the highest value of 0.75 for wind direction $-45^{\circ}-45^{\circ}$ and a minimum value of 0.24 for wind direction $45^{\circ}-135^{\circ}$. The amplitude of the city area has shown a very low correlation both with the wind direction and with the wind speed (correlation factor between amplitude and wind speed: $r=0.2$ for $\mathrm{VH}$ and $r=0.1$ for $\mathrm{VV}$ ).

\subsection{Rain condition}

The rain conditions were also taken into consideration. Over the two years dataset, 25 days with a precipitation accumulation $>0$ at the time of the satellite pass were registered. These days were used to generate scatter plot of the amplitude value (VV and VH polarizations) versus the precipitation accumulations. Despite the low number of days with positive precipitation accumulation at the moment of the satellite pass during the two year analyzed, these data were used to generate scatter plot of the amplitude value (VV and VH polarizations) versus the precipitation accumulations in order to evaluate any possible relation. No particular correlation or trend can be noticed in this analysis for all the considered mask (see Figure 8), and the effect of the rain on the SAR signal is not relevant to get further information on sandy areas.

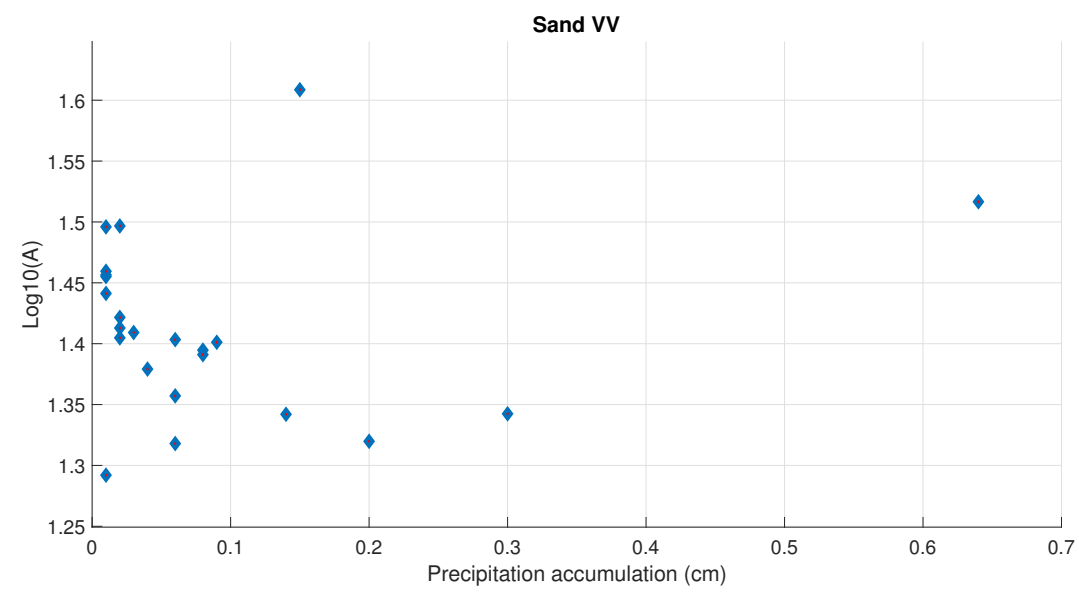

(a)

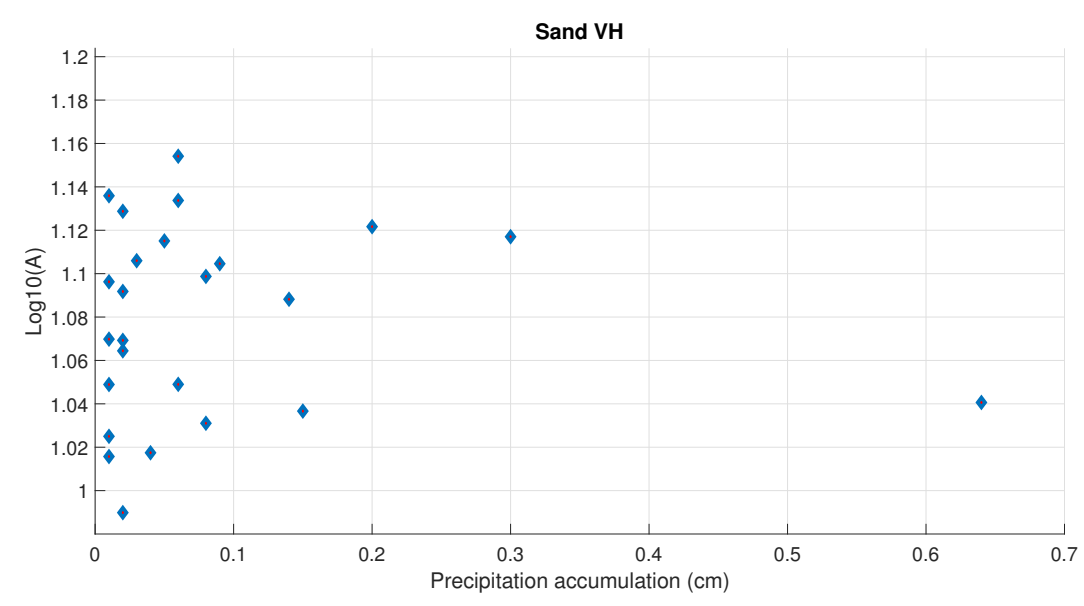

(b)

Figure 8. Scatter plot of amplitude values versus precipitation accumulation evaluated over two years on the sand mask for: (a), VH polarization; and (b), VV polarization. No particular correlation or trend of the amplitude versus precipitation accumulation has be noticed in this analysis. 

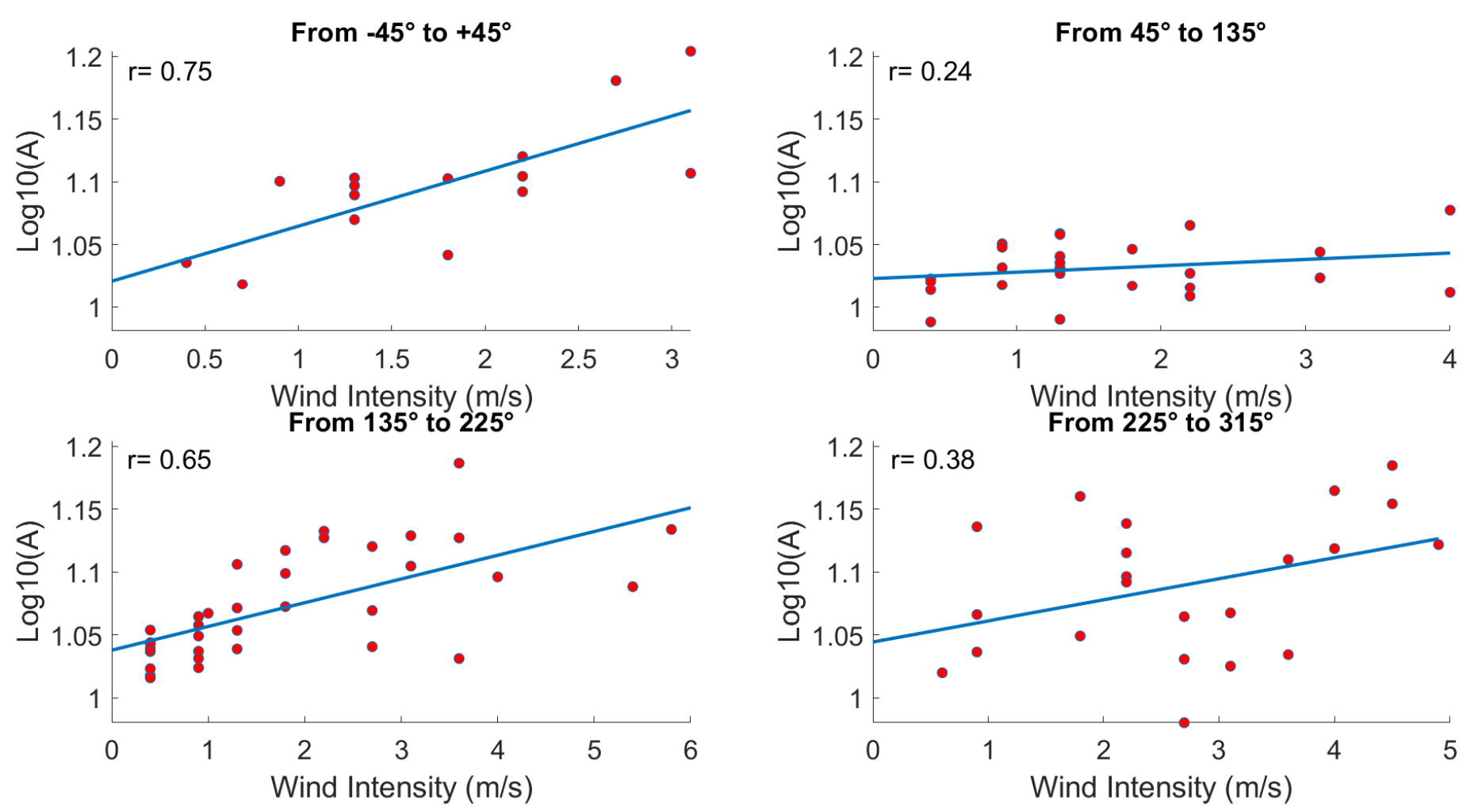

Figure 7. Correlation factor ( $r$ in the figures) between wind amplitude (VH polarization) and wind speed for different wind direction: up left $\left(-45^{\circ}-45^{\circ}\right)$; up right $\left(45^{\circ}-135^{\circ}\right)$; bottom left $\left(135^{\circ}-225^{\circ}\right)$; bottom right $\left(225^{\circ}-315^{\circ}\right)$.

\subsection{Tidal condition analysis}

The sensitivity analysis of the amplitude signal to the tidal condition, which is connected to the sensitivity of the SAR signal to the soil moisture and to the presence of water in the pixels, has been carried out in the following way. The considered area covers a surface of $0.14 \mathrm{~km}^{2}$, and it corresponds to the intertidal mask plus the last part of the dry mask. Sea waves can reach this area in case of very high wind with specific direction. It is reasonable to think that the anthropic influence on this area is particularly low. This area has been divided into 33 transects, $3.5 \mathrm{~m}$ wide, parallel to the waterline. For each transect, the mean multi-temporal amplitude both for VV and VH polarizations has been evaluated (Figure 9). It can be noticed the decreasing trend going from the dry transect $(j=1)$ towards the sea $(j=33)$. Both polarizations show about $6 \%$ decrease of the $\log (A)$ along the transects. The last transects have always either sea water (high tide) or high moisture content (low tide), whilst the water content of the intermediate transects depends on the tide level. When evaluating the correlation of the amplitude for each transect with the tidal condition (Figure 10), it is noticed that there is a sign change. For VH polarization, the correlation with the tide ranges from +0.25 to -0.25 going from the dry transect towards the sea. The correlation factor gets negative with the increase of the water content along the transects (therefore the amplitude decreases). VV polarization seems to have a different sensitivity and the correlation factor is negative for all the analyszed transects. A last plot shows the amplitude variation when separating the low and the high tidal condition (Figure 11). For VH polarization, in case of high tide (higher water level in the transects) the amplitude values are in general lower than the case of low tide (less water content). This does not occur for the transects which are always dry. VV polarization shows the same trend but a different sensitivity and higher amplitude values. This analysis can be further investigated in order to understand to what extent it is possible to detect the water content on the sand considering the sensitivity of the two polarizations. 


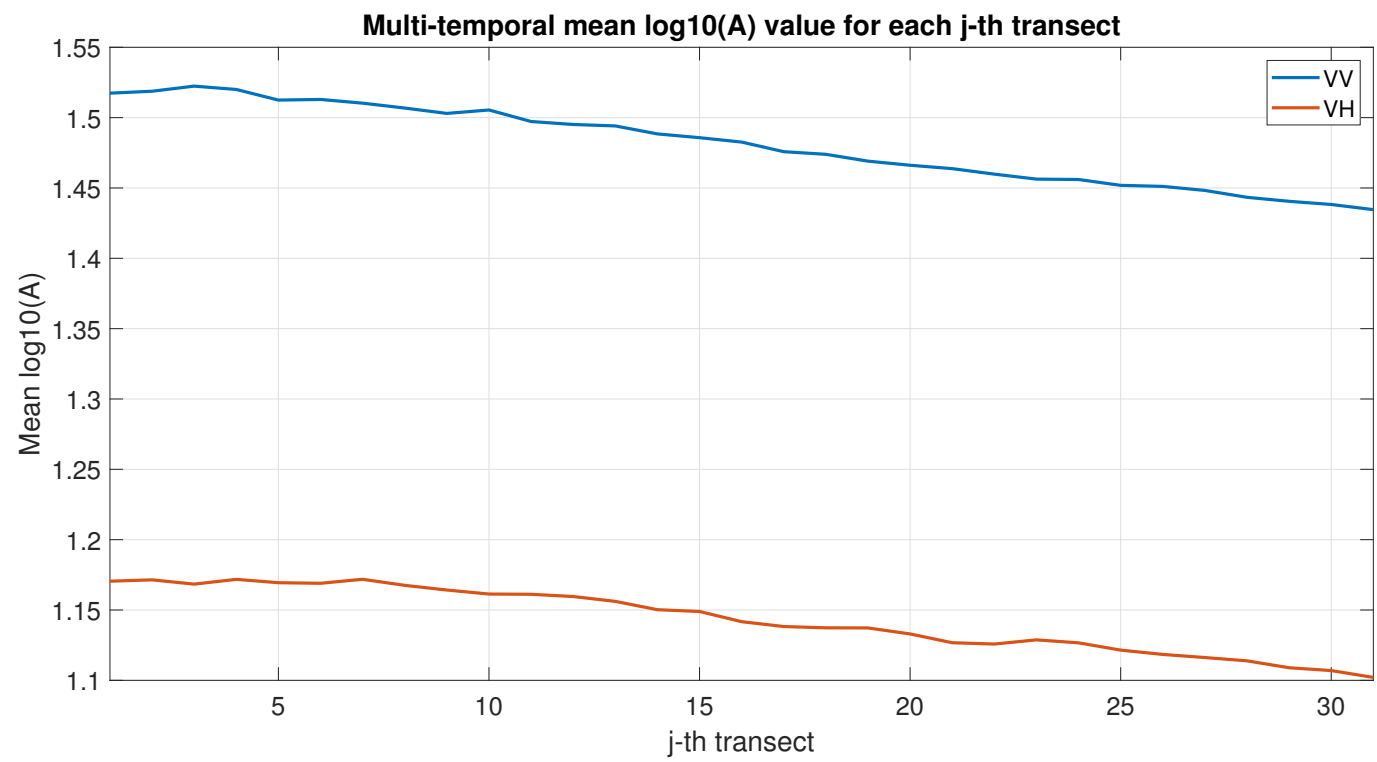

Figure 9. Mean amplitude value (VH and VV polarizations) on the transects parallel to the coastline. A decreasing trend can be noticed for both polarizations moving from the dry transect $(j=1)$ towards the sea $(j=33)$

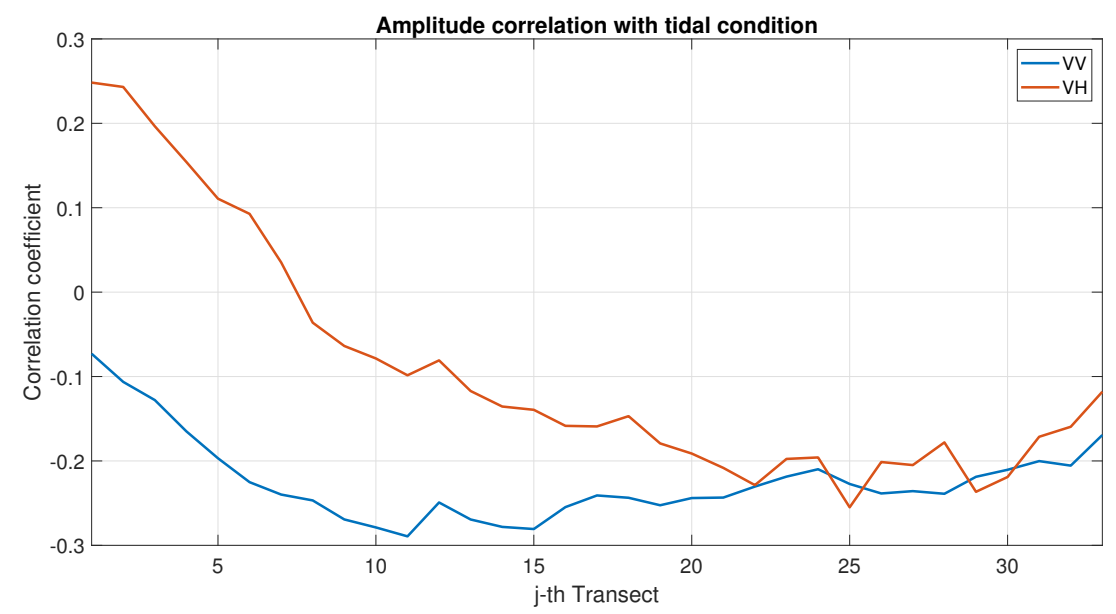

Figure 10. VH and VV Amplitude correlation with tidal conditions 


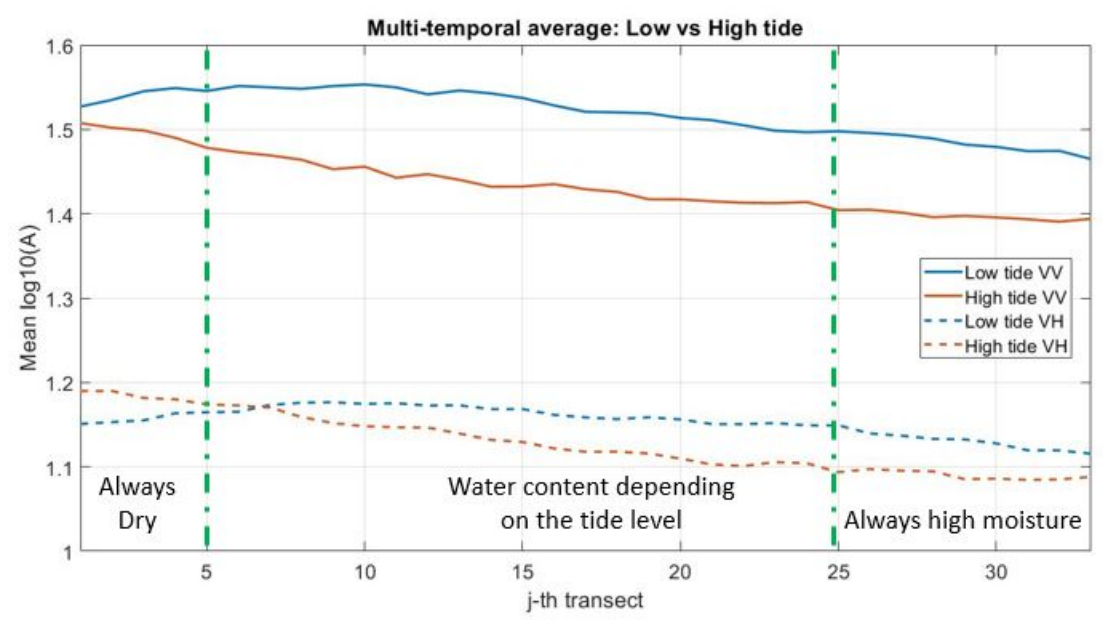

Figure 11. Multi-temporal amplitude mean: Low and high tide trend for VH and VV polarizations. For both polarizations, in case of high tide (higher water level in the transects) the amplitude values are in general lower than the case of low tide (lower water content).

\subsection{Roughness analysis}

The purpose of this section is to evaluate the roughness of the beach surface by creating a digital elevation model (DEM) using a TLS, and evaluating the RMSH for each SAR pixel. These values will be compared to the SAR amplitude. The TLS used for this analysis is a Riegl-VZ2000, which was installed in July 2019 on the upper balcony of a building at $156 \mathrm{~m}$ distance from the beach area of Noordwijk (see Figure 12). It has collected data for two years. The field of view is $\sim 1 \mathrm{~km}$ by $\sim 300 \mathrm{~m}$ and contains a part of the dunes, the beach and the intertidal zone during high and low tide. This yields detailed information on the beach morphology.

\subsubsection{Terrestrial Laser Scanner}

Every hour a dataset containing a 3D representation of the scanned area and the backscatter intensity is collected by the TLS. The Riegl VZ-2000 operates with an accuracy of $8 \mathrm{~mm}$ and a precision of $5 \mathrm{~mm}$ [RIEGL] in the near-infrared. The laser beam divergence is $0.3 \mathrm{mrad}$, and its field of view is $100^{\circ}$ horizontal and $360^{\circ}$ vertical. Each point is associated with a horizontal angle $\phi$ and vertical angle $\theta$. These coordinates are automatically converted into $(x, y, z)$ coordinates relative to the PTLS. The laser scans the area every hour in low resolution, which corresponds to $0.03^{\circ}$ angular point spacing (about $30 \mathrm{~cm}$ point spacing at the water line). Each data point contains $(x, y, z)$ coordinates and reflectance. Low resolution data points, in terms of $(x, y, z)$ coordinates converted to Geographic coordinate system, have been used to perform the relation with SAR data.

\subsubsection{RMSH evaluation}

In order to evaluate the RMSH index over the study area, the following procedure has been adopted:

1. Creation of two different stacks of images. The first stack contains the coregistered Sentinel-1 images, from the same orbit used for the study descripted above, acquired during the period of TLS activity, from July 2019 until April 2020. The second stack contains the coregistered TLS images acquired at the same local time of Sentinel-1 pass over the study area. The two stacks contain 30 images which have been therefore acquired on the same day and at the same local time.

2. TLS and SAR images have been coregistered: each TLS data-point has been associated to the closest SAR pixel center. After the match, at least hundred TLS data-points are 


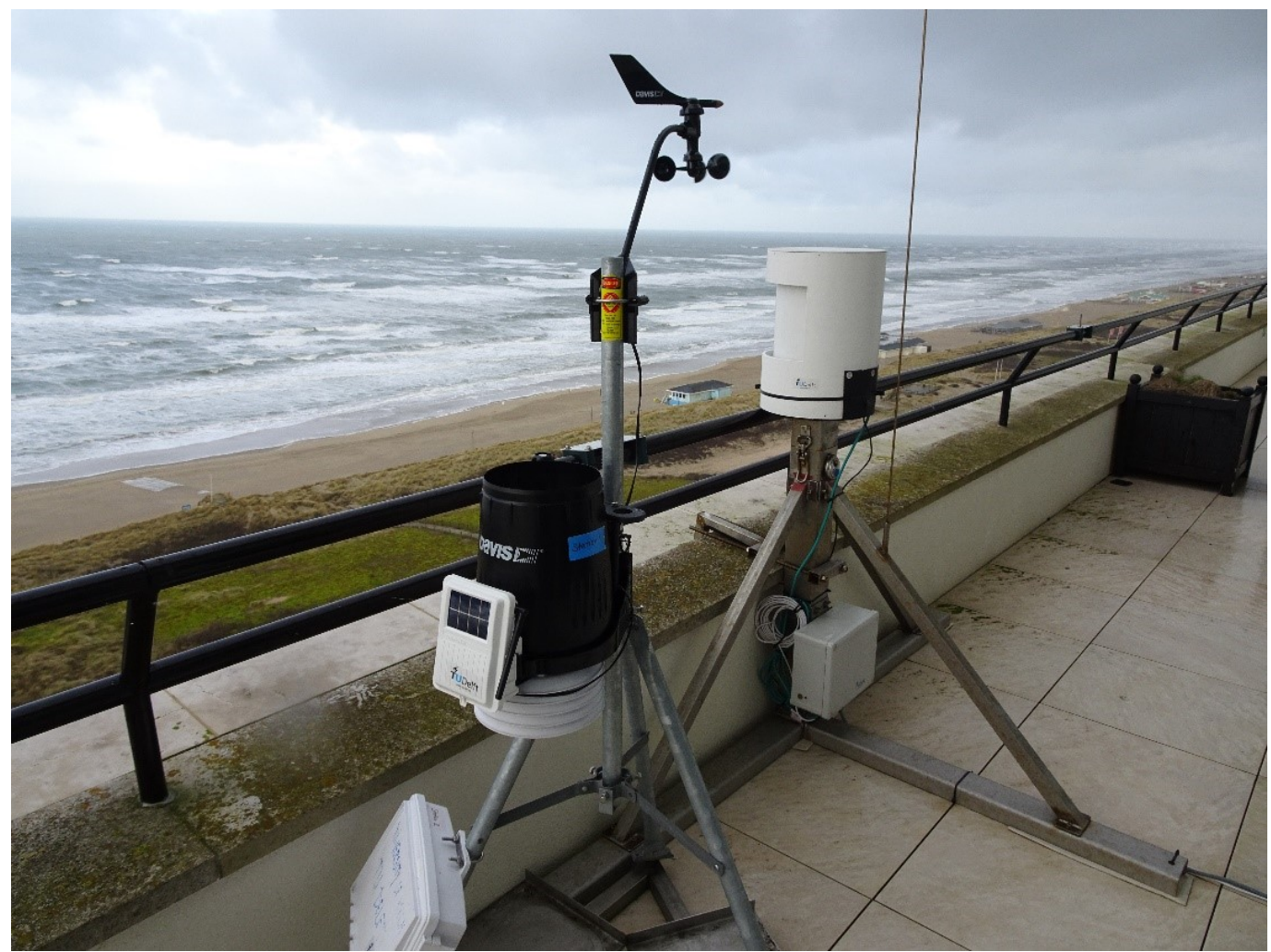

Figure 12. TLS and weather station installed on the upper balcony of the Huis ter Duin Hotel, Noordwijk, the Netherlands

associated to each SAR pixel. SAR pixels with a low number of TLS data-points have not been considered.

3. The $z$ values of the data points belonging to each SAR pixel have been interpolated on a $M \times N$ pixels grid (with resolution of $0.1 \mathrm{e}-5$ degrees in latitude and longitude) creating a local DEM for each SAR pixel. For each grid, RMSH has been evaluated as in [haubrock2009spatiotemporal]:

$$
\mathrm{RMSH}=\sqrt{\frac{1}{M N} \sum_{c=0}^{M-1} \sum_{r=0}^{N-1}\left[z\left(x_{c}, y_{r}\right)-\mu\right]^{2}}
$$

where:

$M=$ number of columns;

$N=$ number of rows;

$c=$ column index;

$r=$ row index;

$z\left(x_{c}, y_{r}\right)=z$-value at position $x_{c}, y_{r}$;

$\mu=$ average $z$-value

4. Using (1) each SAR pixel is characterized by its own local RMSH value, with a $\sim 10 \mathrm{~cm}$ resolution.

\subsubsection{RMSH correlation}

In total, $\sim 400$ pixels have been considered in the present analysis. For each SAR pixel, a time-serie has been developed containing RMSH values as a funcion of $\log _{10}(A)$, and their correlation has been evaluated. The correlation maps between RMSH values and $\log _{10}(A)$ (Figure 13 (left)) have been compared to the mean RMSH for each pixel (Figure 13 (right)). Figure 13 (right) shows how, excluding the upper right area corresponding to the 
end of the dunes area, RMSH values are almost constant over the beach, and that they get lower moving toward the sea. Figure 13 (left) shows how the range of the correlation values is very wide (from -0.65 up to +0.65 ). No evident correlation seems to exist between RMSH values and correlation values, and that the relation between $\mathrm{RMSH}$ and $\log _{10}(A)$ could have a stochastic nature.
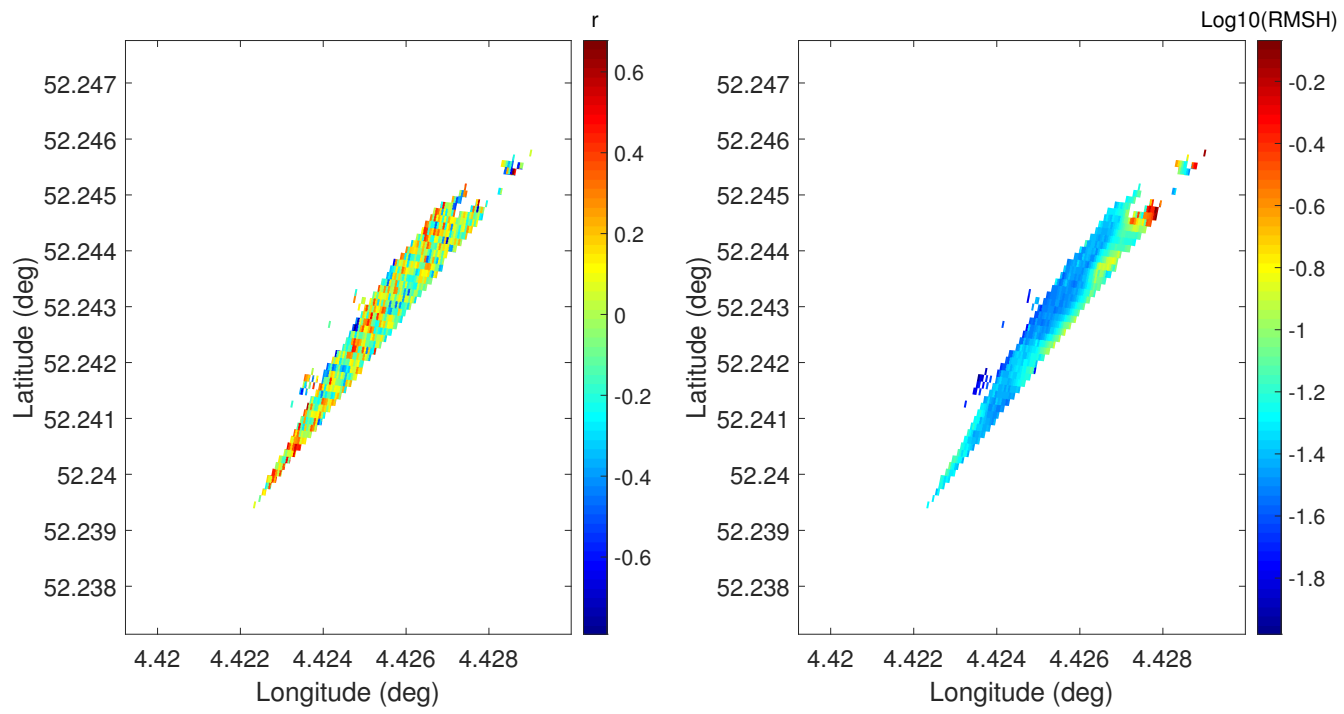

Figure 13. Correlation map between RMSH values and $\log _{10}(A)$ for each pixel of the study area (left panel); and mean RMSH value for each pixel of the study area (right panel)

\section{Conclusions}

This paper presents the results of a feasibility study intended to gain insight into the potential and limitations of SAR imagery for the retrieval of soil properties over coastal areas. The most relevant result is a strong correlation between the amplitude evaluated on the sandy area, both for VV and VH polarizations, and the local wind. The distribution of wind intensity is associated to low or high amplitude depending on the wind direction, and the correlation factor between amplitude and wind intensity significantly increases or decreases when focusing the analysis to specific wind direction. On the other hand, mean RMSH analysis showed that no evident correlation seems to exist between roughness and amplitude. Considering these phenomena, the hypothesis is that specific wind direction causes a surface roughness variation on the sand which gives an higher correlation with the backscatter signal, compared to other wind direction. The rain conditions were taken into consideration considering the precipitation accumulation on the beach of Noordwijk at the moment of the satellite pass, and no particular correlation or trend of the amplitude could be noticed. The sensitivity analysis of the amplitude signal to the tidal condition has been carried out considering transects of the beach parallel to the coastline in the intertidal area. Both polarizations show a decreasing trend of the amplitude along the transects, moving from the dry area of the beach towards the sea. The correlation coefficient between the amplitude and the tide level gets negative with the increase of the water content along the transects.

Future work will include the creation of a code which, by using SAR data as an input, will provide (i) a deeper knowledge of causal empirical relationship between weather phenomena and protective capability of beaches; (ii) classification maps of the coastal environment (shoreline position, intertidal/dune area) which, thanks to a long-term analysis, will allow the understanding of their multitemporal evolution depending on natural and anthropic phenomena. (iii) a deeper investigation about the different contribute of moisture and roughness on the amplitude signal. 


\section{Declaration of competing interest}

The authors declare that they have no known competing financial interests of personal relationships that could have appeared to influence the work reported in this paper.

\section{Acknowledgments}

For the present work, VDB and RH were supported by Mitsubishi Electric Corporation via the project Detecting, Identifying, and Classifying Sandy Soils using Satellite SAR Data. The pre-processing of the data has been performed with the SNAP tool provided by ESA at the website: https://step.esa.int/main/toolboxes/snap/. 
